\title{
Permanência de dente canino decíduo em gato-do-mato-pequeno (Leopardus guttulus)
}

\author{
[Persistence of canine deciduous teeth in a little spotted cat (Leopardus guttulus)]
}

\section{"Relato de Caso/Case Report"}

\section{Caio Henrique de Oliveira Carniatto ${ }^{1 *}$, César Vinícius Gil Braz do Prado ${ }^{2}$, Fernando Lunardelli ${ }^{3}$, Ana Paula Vidotti ${ }^{4}$}

\author{
1Programa de Pós-Graduação em Ciências Veterinárias, Universidade Federal do Paraná, Curitiba/PR, Brasil \\ ${ }^{2}$ Médico veterinário autônomo, São Paulo/SP, Brasil \\ ${ }^{3}$ Centro de Diagnóstico Veterinário (CDVET), Maringá/PR, Brasil \\ ${ }^{4}$ Departamento de Ciências Morfológicas da Universidade Estadual de Maringá - DCM/UEM, Maringá/PR, Brasil \\ *Autor para correspondência: E-mail: caiocarniatto@gmail.com
}

\section{Resumo}

Malformações dentárias são comuns em mamíferos silvestres, entretanto, são pouco documentadas na literatura científica. Nesse sentido, o conhecimento das condições patológicas dentárias em espécies de vida livre é fundamental para a medicina veterinária, permitindo o diagnóstico e tratamento destas enfermidades. $\mathrm{O}$ objetivo deste estudo foi relatar a persistência de dente canino decíduo em gato-do-mato-pequeno (Leopardus guttulus), doado pela polícia militar ambiental ao Laboratório de Zoologia do Centro Universitário Cesumar - UniCesumar, Maringá, Paraná, Brasil. Analisou-se o crânio de um felino macho (comprimento da face: $2 \mathrm{~cm}$; comprimento do crânio: $6,3 \mathrm{~cm}$; comprimento total do crânio: 7,5cm; largura da face: 4,5cm; largura do crânio: $5,3 \mathrm{~cm}$ ). Observando a dentição e discretas fusões de suturas cranianas, foi estimado que era um animal juvenil. O espécime apresentou persistência dos dentes caninos decíduos maxilares direito e esquerdo, localizados caudalmente aos caninos permanentes. Ambos os caninos decíduos mandibulares apresentaram uma fenda na coroa, que não se estendeu à região da raiz dentária. Os caninos decíduos e permanentes maxilares direito e esquerdo ocupavam o mesmo alvéolo. Os pré-molares decíduos maxilares estavam projetados, mas não os mandibulares, assim como os molares maxilares e mandibulares. Devido à falta de informações sobre a idade em que os dentes decíduos esfoliam e os definitivos sofrem erupção em L. guttulus e em outras espécies brasileiras, estudos devem ser conduzidos em filhotes mantidos em cativeiro, visando avaliar enfermidades orais e dentais e como estas se manifestam em felinos silvestres.

Palavras-chave: felino; odontologia veterinária; malformação.

\begin{abstract}
Dental malformations are common in wild mammals, however, they are poorly documented in the scientific literature. Knowledge of dental pathological conditions in free ranging species is fundamental for veterinary medicine, allowing the diagnosis and treatment of these diseases. The objective of this study was to report the persistence of a deciduous canine tooth in a little spotted cat (Leopardus guttulus), donated by the Environmental Police to the Laboratory of Zoology of Centro Universitário Cesumar - UniCesumar, Maringá, Paraná State, Brazil. The skull of a male was analyzed (face length: $2 \mathrm{~cm}$; skull length: $6.3 \mathrm{~cm}$; total length of the skull: $7.5 \mathrm{~cm}$; face width: $4.5 \mathrm{~cm}$; skull width: $5.3 \mathrm{~cm}$ ). Observing the dentition and discrete fusions of cranial sutures, the age of the animal was estimated between six and seven months, considered a juvenile animal. The specimen showed persistence of the right and left deciduous canine teeth, located caudal to the permanent canines. Both mandibular deciduous canines presented a crack in the crown, which did not extend to the region of the dental root. The right and left maxillary deciduous and permanent canines occupied the same alveolus. The primary maxillary premolars were designed, but not the mandibular, as well as the maxillary and mandibular molars. Due to the lack of information about the age at which deciduous teeth exfoliate and the definitive ones erupt in L. guttulus and other Brazilian species, studies should be conducted on kittens kept in captivity, aiming to evaluate oral and dental pathologies and how they manifest themselves in wild cats.
\end{abstract}

Keywords: feline; malformation; veterinary dentistry.

Recebido 02 de fevereiro de 2019. Aceito 08 de fevereiro de 2021.

DOI: https://doi.org/10.26605/medvet-v15n1-2374 


\section{Introdução}

Entre os vertebrados, principalmente em espécies terrestres, a classe Mammalia é o grupo que apresenta maior variedade morfológica e funcional dos dentes. A maioria das espécies é classificada como difiodonte, apresentando dois conjuntos de dentições, a decídua e a definitiva. $\mathrm{O}$ desenvolvimento dos dentes, na maioria das espécies, ocorre após a amamentação, e os mamíferos, com raras exceções, apresentam quatro tipos de dentes: incisivos, caninos, prémolares e molares; os únicos dentes que não são substituídos são os molares, os demais são decíduos (Van Nievelt e Smith, 2005; Pough et al., 2008; Liem et al., 2012).

Os incisivos são dentes pequenos e estão localizados cranialmente aos demais, podendo ser cônicos ou laminados, adaptados a segurar o alimento na cavidade oral. Os caninos são dentes simples, em formato de espinho, adaptados a perfurar as presas e rasgar carnes, assim como participar de lutas e outros comportamentos sociais. Os dentes de substituição, caudais aos caninos, são pré-molares, seguidos pelos molares, dentes não-decíduos (Hickman et al., 2016). Os dentes pré-molares e molares são os mais variáveis nos mamíferos. Enquanto os pré-molares puncionam o alimento, os molares têm a função de cortar e esmagar o conteúdo alimentar. Em carnívoros, o quarto pré-molar superior, associado ao primeiro molar inferior, compõe o aparelho carniceiro, promovendo uma adaptação eficiente no corte de carne (Liem et al., 2012).

A ordem Carnivora é subdividida nas subordens Feliformia e Caniformia, e é composta pelos mamíferos carnívoros, terrestres e semiaquáticos (Fregonezi et al., 2015). No Brasil, a família Felidae é representada por três gêneros e oito espécies, quase todas ameaçadas de extinção. A espécie Leopardus guttulus, popularmente conhecida como gato-do-mato, é uma espécie pouco estudada, considerada a menor espécie felina brasileira, com anatomia similar à do gatodoméstico (Cheida et al., 2006; Silva e Adania, 2006; Carvalho et al., 2007; De Bestiani et al., 2015).

Doenças orais e dentais são os diagnósticos mais comuns em gatos domésticos (Wiggs e Bloom, 2011; Howes et al., 2019; Silva et al., 2019), mas pouco se sabe sobre a manifestação de doenças orais em felinos silvestres. A permanência de dentes decíduos, em carnívoros, é uma afecção relativamente comum (Legendre,
1995), e é caracterizada pela retenção de dente decíduo, que prejudica ou impede a projeção do dente definitivo.

Em carnívoros, a retenção do dente decíduo pode causar dor e desconforto, além de maloclusões, traumas na gengiva, mordidas irregulares, periodontites e processos inflamatórios (Hock, 1975; Amimoto et al., 1993; Gioso e Carvalho, 2003; Boy et al., 2016). A retenção pode ser consequência de discrepâncias no crescimento dos ossos mandibulares e dos próprios dentes (Polkowska et al., 2014). Como prejudica a projeção do dente definitivo, o dente decíduo permanecente deve ser extraído o quanto antes (Fulton et al., 2014).

Embora a fisiopatologia de doenças orais em carnívoros domésticos é constantemente pesquisada e monitorada, ainda há uma lacuna no conhecimento destas doenças em felinos silvestres, de vida livre ou mantidos em zoológicos. Nesse sentido, reportamos um caso de permanência de caninos decíduos em um macho de Leopardus guttulus.

\section{Descrição do Caso}

O corpo de um macho de L. guttulus, em bom estado de conservação e vítima de atropelamento, foi doado pela polícia militar ambiental ao Laboratório de Zoologia do Centro Universitário Cesumar - Unicesumar, em Maringá, Paraná, Brasil.

O crânio foi removido, macerado e branqueado segundo técnica descrita por Hildebrand e Goslow Jr. (2006), e os órgãos internos fixados em formol para futuros estudos. $\mathrm{O}$ corpo e a pele foram preparados para taxidermia. As medições morfométricas do crânio seguiram Gomes et al. (2015). A idade estimada do animal foi baseada em Nascimento (2014), onde foram observadas a presença de dentes decíduos e permanentes e a fusão de suturas cranianas. Os exames radiográficos foram realizados em uma clínica veterinária, através de projeções laterais e ventrodorsais.

Após preparação osteológica, o crânio apresentou as seguintes medidas biométricas: comprimento da face: $2 \mathrm{~cm}$; comprimento do crânio: $6,3 \mathrm{~cm}$; comprimento total do crânio: $7,5 \mathrm{~cm}$; largura da face: $4,5 \mathrm{~cm}$; largura do crânio: $5,3 \mathrm{~cm}$. Observando a dentição e discretas fusões de suturas cranianas, a idade do animal foi estimada entre seis e sete meses, sendo considerado um animal juvenil. 
O espécime apresentou persistência dos dentes caninos decíduos maxilares direito e esquerdo, localizados caudalmente aos caninos permanentes (Figuras 1A e 1B). Ambos caninos decíduos mandibulares apresentaram uma fenda na coroa (Figura 1C), que não se estendeu à região da raiz dentária. Os caninos decíduos e permanentes maxilares direito e esquerdo ocupavam o mesmo alvéolo. Já os pré-molares decíduos maxilares estavam projetados, mas não os mandibulares, assim como os molares maxilares e mandibulares (Figuras 2A e 2B).
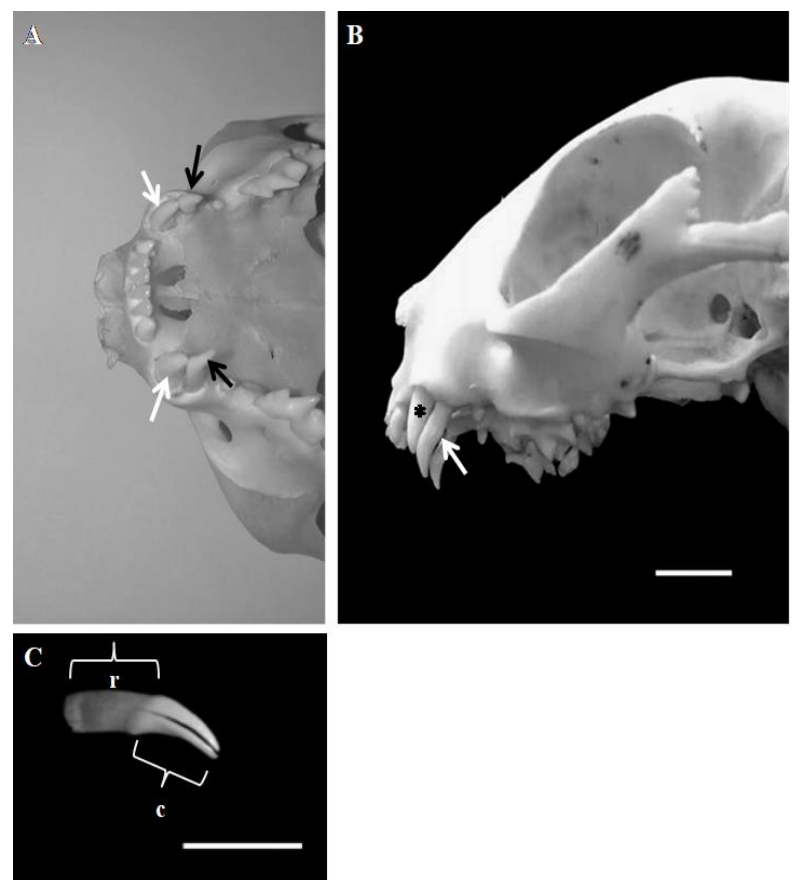

Figura 1. A) Fotomacrografia ventral do crânio de gato-do-mato-pequeno (Leopardus guttulus). As setas brancas apontam os caninos permanentes. As setas pretas indicam os caninos decíduos. B) Fotomacrografia lateral esquerda do crânio. Notar o canino decíduo (seta) localizado caudalmente ao canino definitivo (*), ambos ocupando o mesmo alvéolo. C) Fotomacrografia lateral esquerda do canino decíduo mandibular direito. Notar a fissura na coroa (c) e a raiz (r) do dente. Barra: $1 \mathrm{~cm}$.

\section{Discussão}

A persistência de dentes decíduos é frequente em animais silvestres (Gioso et al., 2014), porém, a documentação de alterações dentárias em animais de vida livre é rara e esporádica. Alterações dentárias em mamíferos, como dentes supranuméricos ou ausentes e retenção de dentes decíduos são relatadas em leões-marinhos (Drehmer et al., 2004, Drehmer et al., 2009), lobos-marinhos (Drehmer e Ferigolo,
1996), cachorros-do-mato (Rossi Junior et al., 2013), morcegos (Rui e Drehmer, 2004), gambás (De Moraes et al., 2001), mão-pelada (Silva e Quintela, 2011; Bianchi et al., 2013), cachorros (Gaspar e Amaral, 1995; Lacerda et al., 2000), cavalos (Dixon et al., 2005) e gatos (Gawor e Niemiec, 2014; Langley-Hobbs et al., 2015). Aghashani et al. (2016), estudando crânios de lince-pardo (Lynx rufus californicus), identificou a persistência de três incisivos mandibulares em duzentos e setenta e sete crânios analisados, sendo um crânio com dois dentes persistentes e um crânio com apenas um dente persistente.
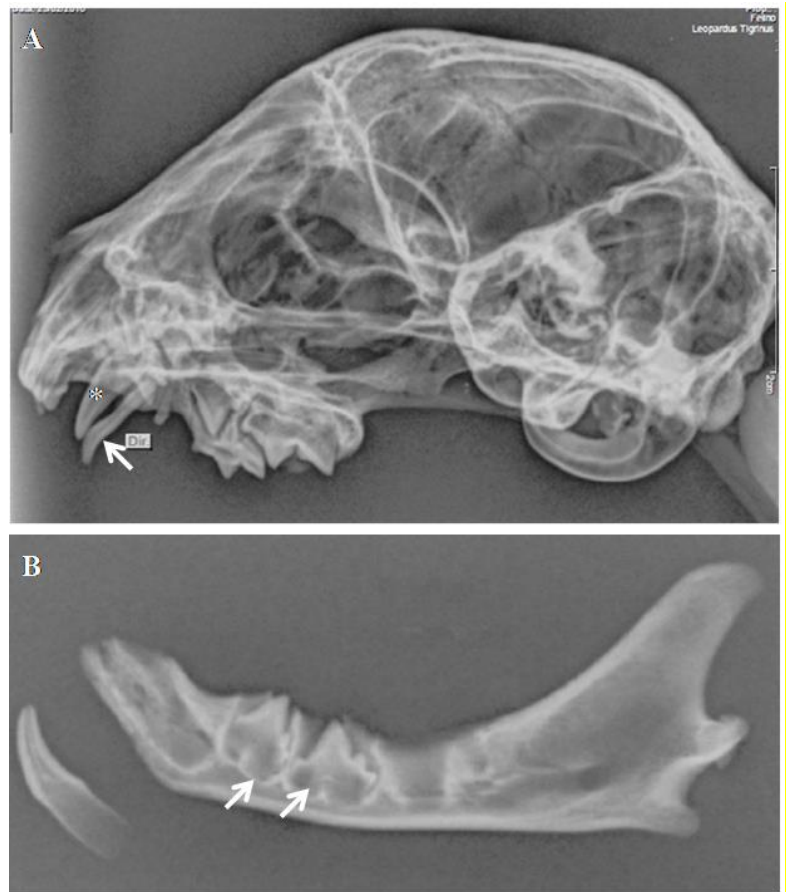

Figura 2. Radiografia lateral esquerda do crânio de gato-do-mato-pequeno

(Leopardus guttulus). O dente decíduo (seta) ocupa a maior parte do alvéolo, prejudicando a projeção do canino definitivo (*) (A). Radiografia lateral esquerda da mandíbula. Notar a projeção dos pré-molares (setas) (B).

Enfermidades orais e dentárias são os diagnósticos mais comuns na clínica de carnívoros domésticos (Pachaly et al., 2010; Wiggs e Bloom, 2011), decorrentes de alterações complexas e multifatoriais, como influência genética, morfologia craniana e dieta (Marker e Dickman, 2004), podendo influenciar na queda do dente decíduo (DuPont e DeBowes, 2009) e na projeção dos dentes permanentes (Teixeira et al., 2005). No entanto, para que estudos sobre o impacto e prevalência de anomalias orais em carnívoros silvestres sejam realizados, há a necessidade de 
que estas sejam definidas, caracterizadas e descritas (Marker e Dickman, 2004).

A queda dos dentes decíduos ocorre quando há reabsorção radicular (rizólise), com a redução do comprimento das raízes dentárias, processo conhecido como esfoliação (Rossi e Rossi, 2010). No crânio estudado não foi observado processo de rizólise, não sendo possível estimar o grau de reabsorção dos dentes decíduos. Neste crânio os molares mandibulares não estavam projetados; no gato doméstico (Felis silvestris catus) e no gatosilvestre-europeu (Felis silvestris), a projeção dos molares ocorre antes do quarto ou quinto mês (Roza, 2004), podendo se estender até o sétimo (König et al., 2011; Stefen e Heidecke, 2012).

No animal estudado os dentes decíduos estavam localizados caudalmente aos dentes definitivos, ao contrário do que é relatado por Hoskins (1992) em gatos domésticos, onde geralmente o dente decíduo está localizado lateralmente ao dente permanente. A posição dos dentes decíduos corrobora com os achados em filhotes de Puma concolor, com a permanência de caninos decíduos maxilares e mandibulares em deslocamentos caudais, e em cães domésticos (Rankin, 2011; Santos et al., 2014).

A permanência dos dentes decíduos em animais de vida livre pode prejudicar atividades como caça e alimentação, ocasionando uma desvantagem entre o animal e seus competidores ou predadores. Em animais domésticos ou silvestres mantidos em cativeiro, recomenda-se a extração dos dentes decíduos (Tutt, 2006).

Em estudos com onças-pintadas (Panthera onca) cativas e de vida livre, Junior et al. (2007) afirmam que é difícil estimar se achados clínicos decorrentes de doenças orais e bucais, como placa bacteriana e gengivite, são significativos, pois os animais podem vir à óbito antes das manifestações clínicas.

Devido à falta de informações sobre a idade em que os dentes decíduos esfoliam e os definitivos sofrem erupção em L. guttulus e em outras espécies brasileiras, estudos devem ser conduzidos em filhotes mantidos em cativeiro, visando avaliar enfermidades orais e dentais e como estas se manifestam em felinos silvestres.

\section{Conflito de interesse}

Os autores declaram não existir conflito de interesse.

\section{Referências}

Aghashani, A.; Kim, A.S.; Kass, P.H.; Verstraete, F.J.M. Dental pathology of the California bobcat (Lynx rufus californicus). Journal of Comparative Pathology, 154 (4): 329-340, 2016.

Amimoto, A.; Iwamoto, S.; Taura, Y.; Nakama, S.; Yamanouchi, T. Effects of surgical orthodontic treatment for malalignment due to the prolonged retention of deciduous canines in young dogs. Journal of Veterinary Medical Science, 55(1): 73-79, 1993.

Bianchi, M.A.F.; Mayorga, L.F.S.P.; Castro, A.P.A.; Rossi Junior, J.L. Estudo das alterações ante-morte da cavidade oral de mãos-peladas (Procyon cancrivorus) de vida livre e de cativeiro. Pesquisa Veterinária Brasileira, 33 (5): 651-661, 2013.

Boy, S.; Crossley, D.; Steenkamp, G. Developmental structural tooth defects in dogs - experience from veterinary dental referral practice and review of the literature. Frontiers in Veterinary Science, 3: 1-13, 2016.

Carvalho, P.S.L.; Pereira, G.G.; Petrus, L.C.; Soares, E.C.; Michima, L.E.; Larsson, M.H.M.A. Avaliação de alguns parâmetros ecocardiográficos do gato-do-mato (Leopardus tigrinus), mantido em cativeiro e submetido à anestesia com xilazina e quetamina. Arquivo Brasileiro de Medicina Veterinária e Zootecnia, 59 (3): 695-699, 2007.

Cheida, C.C.; Nakano-Oliveira, E.; Fusco-Costa, R.; Rocha-Mendes, F.; Quadros, J. Ordem Carnivora. In:__ Reis, N.R.; Peracchi, A.L.; Pedro, W.A. Lima I.P. Mamíferos brasileiros. Londrina: Universidade Estadual de Londrina, 2006. p. 231-276.

De Bestiani, E.; Bazilio, S.; De Barros, K.F.; Nabrzecki, G. Felinos da floresta nacional de Piraí do Sul, Paraná - Brasil. Acta Zoológica Mexicana, 31: 23-26, 2015.

De Moraes, D.A.; Lemos, B.; Cerqueira, R. Supernumerary molars in neotropical opossums (Didelphimorphia, Didelphidae). Mammalian Biology, 66: 193-203, 2001.

Dixon, P.M.; Easley, J.; Ekmann, A. Supernumerary teeth in the horse. Clinical Techniques in Equine Practice, 4(2): 155161, 2005.

Drehmer, C.J.; Ferigolo, J. Anomalias e patologias dentárias em Arctocephalus G. Saint-Hilaire \& Cuvier (Pinnipedia, Otariidae) 
da costa do Rio Grande do Sul, Brasil. Revista Brasileira de Zoologia, 13: 857-865, 1996.

Drehmer, C.J.; Fabián, M.E.; Menegheti, J.O. Dental anomalies in the Atlantic population of South America sea lion, Otaria Byronia (Pinnipedia, Otariidae): evolutionary implications and ecological approach. Latin American Journal of Aquatic Mammals, 3: 7-18, 2004.

Drehmer, C.J.; Dornelles, J.E.; Loch, C. Variações na fórmula dentária de Otaria byronia Blainville (Pinnipedia, Otariidae) no Pacífico: registro de um novo tipo de anomalia. Neotropical Biology and Conservation, 4 (1): 28-35, 2009.

DuPont, G.A.; DeBowes, L.J. Atlas of dental radiography in dogs and cats. St. Louis: Saunders Elsevier, 2009. 288p.

Fregonezi, M.N.; Reis, N.R.; Peracchi, A. Classe Mammalia. In: Benedito, E. Biologia e ecologia dos vertebrados. Rio de Janeiro: Roca, 2015. p. 213-224.

Fulton, A.J.; Fiani, N.; Verstraete, F.J.M. Canine pediatric dentistry. Veterinary Clinics: Small Animal Practice, 44: 303-324, 2014.

Gaspar, L.F.J.; Amaral, A.S. Nanismo hipofisário em um canino: achados clínicos e laboratoriais. Ciência Rural, 25 (3): 465-468, 1995.

Gawor, J.; Niemiec, B.A. Oral pathology in dog and cat: overview of selected problems. The Veterinary Nurse, 5(1): 10-18, 2014.

Gioso, M.A.; Carvalho, V.G.G. Maxillary dentigerous cyst in a cat. Journal of Veterinary Dentistry, 20(1): 28-30, 2003.

Gioso, M.A.; Fecchio, R.S.; Martinez L.A.V. Radiologia odontológica. In: Cubas, Z.S.; Silva, J.C.R.; Catão-Dias, J.L. Tratado de animais selvagens: medicina veterinária. $2^{\mathrm{a}}$ ed. São Paulo: Roca, 2014. p. 1693-1782.

Gomes, M.S.; Moraes, S.O.S.; Sousa, C.A.S.; Souza Junior, P.; Figueiredo, M.A. Craniometria, morfometria do encéfalo e tipologia do circuito arterial da base do encéfalo em gatos. Revista Brasileira de Ciência Veterinária, 22: 3-9, 2015.

Hickman, C.P.; Roberts, L.S.; Keen, S.S.; Eisenhour, D.J.; Larson, A.; L'Anson, H. Princípios integrados de zoologia. $16^{\mathrm{a}} \mathrm{ed}$. Rio de Janeiro: Guanabara Koogan, 2016. 937p.

Hildebrand, M.; Goslow Jr., G.E. Análise da estrutura dos vertebrados. $2^{\text {a }}$ ed. São Paulo: Atheneu, 2006. 637p.
Hock, J. Gingival vasculature around erupting deciduous teeth of dogs and cats. Journal of Clinical Periodontology, 2(1): 44-50, 1975.

Hoskins, J.D. Pediatria veterinária: cães e gatos até 6 meses de idade. São Paulo: Manole, 1992. 605p.

Howes, C.; Longley, M.; Reyes, N.; Major, A.C.; Gracis, M.; Scanlan, A.F.; Bailey, S.; LangleyHobbs, S.J. Skull pathology in 10 cats with patelar fracture and dental anomaly syndrome. Journal of Feline Medicine and Surgery, 21(8): 793-800, 2019.

Junior, J.L.R.; Gioso, M.A.; DominguesFalqueiro, L.M. Estudo comparativo sobre prevalência de doença periodontal em Panthera onca mantida em cativeiro e em indivíduos de natureza. Pesquisa Veterinária Brasileira, 27: 209-214, 2007.

König, H.E.; Sótonyi, P.; Liebich, H.-G. Sistema digestório (apparatus digestorius). In: König, H.E.; Liebich, H.-G. Anatomia dos animais domésticos. $4^{\text {a }}$ ed. Porto Alegre: Artmed, 2011. p. 321-387.

Lacerda, M.S.; Oliveira, S.T.; Queiroz, D.N. Variações anatômicas na dentição de cães sem raça definida. Ciência Rural, 30: 655-659, 2000.

Langley-Hobbs, S.J.; Longley, M.; Gracis, M. Retained and persistent deciduous teeth in cats. The Veterinary Record, 176(4): 106, 2015.

Legendre, L.F.J. Dentistry on deciduous teeth: what, when, and how. Canadian Veterinary Journal, 35: 793-794, 1995.

Liem, K.F.; Bemis, W.E.; Walker Jr, W.F.; Grande, L. Anatomia funcional dos vertebrados: uma perspectiva evolutiva. São Paulo: Cengage Learning, 2012. 705p.

Marker, L.L.; Dickman, A.J. Dental anomalies and incidence of palatal erosion in Namibian cheetahs (Acinonyx jubatus jubatus). Journal of Mammalogy, 85 (1): 19-24, 2004.

Nascimento, F.O. On the morphological variation and taxonomy of the Geoffroy's cat Leopardus geoffroyi (d'Orbigny \& Gervais, 1844) (Carnivora, Felidae). Papéis Avulsos de Zoologia, 54 (11): 129-160, 2014.

Pachaly, J.R.; Javorouski, M.L.; Koproski, L.; Prazeres, R.F.; Kieras Junior, A. Procedimentos de exodontia em uma lontra (Lontra longicaudis Olfers, 1818). Medvep Revista Científica de Medicina Veterinária Pequenos Animais e Animais de Estimação, 8 (25): 280-283, 2010. 
Polkowska, I.; Gołyńska, M.; Sobczyńska-Rak, A.; Putowska, K.; Matthews-Brzozowska, T.; Szyszkowska, A.; Gołyński, M.; Chełmiński, A.; Capik, I. Orthodontic treatment of dogs on the basis of modern knowledge and own experience. Bulletin of the Veterinary Institute in Pulawy, 58: 645-650, 2014.

Pough, F.H.; Janis, C.M.; Heiser, J.B. A vida dos vertebrados. $4^{\mathrm{a}}$ ed. São Paulo: Atheneu, 2008. $684 p$.

Rankin, K. Pediatric dental abnormalities. Veterinary Technician, 32: 1-8, 2011.

Rossi, A.; Rossi, M. Mecanismos celulares e moleculares envolvidos na reabsorção radicular fisiológica de dentes decíduos. Pesquisa Brasileira em Odontopediatria e Clínica Integrada, 10: 505-511, 2010.

Rossi Junior, J.L.; Castro, A.P.A.; Marchesi, M.D. Avaliação das alterações odontológicas em sincrânios de Cerdocyon thous oriundos de atropelamentos na rodovia ES-060, Espírito Santo. Pesquisa Veterinária Brasileira, 33(6): 785-790, 2013.

Roza, M.R. Odontologia em pequenos animais. Rio de Janeiro: L. F. Livros de Veterinária, 2004. 361p.

Rui, A.M.; Drehmer, C.J. Anomalias e variações na fórmula dentária em morcegos do gênero Artibeus Leach (Chiroptera, Phyllostomidae). Revista Brasileira de Zoologia, 21: 639-648, 2004.

Santos, I.F.C.; Bene, M.; Gaspar, B.; Bambo, O.; Cardoso, J.M.M. Persistência de dentes decíduos em cão (Canis familiares): relato de caso. Revista Científica da Universidade Eduardo Mondlane, Série: Ciências Agronômicas, Florestais e Veterinárias, 1: 75-81, 2014.
Silva, J.C.R.; Adania, C.H. Carnivora - Felidae (onça, suçuarana, jaguatirica, gato-do-mato). In: Cubas, Z.S.; Silva, J.C.R.; Catão-Dias, J.L. Tratado de animais selvagens: medicina veterinária. $1^{a}$ ed. São Paulo: Roca, 2006. p. 505-546.

Silva, R.Z.; Quintela, F.M. Anormalidades dentárias em Procyon cancrivorus (Carnivora, Procyonidae): relato de caso. Estudos de Biologia, 33: 119-122, 2011.

Silva, A.P.; Flores, M.; Mazaro, R.; Luz, F.; Silva, M.; Fighera, R.A. Afecções orais e retroviroses em gatos de abrigo. Pesquisa Veterinária Brasileira, 39(7): 516-522, 2019.

Stefen, C.; Heidecke, D. Ontogenetic changes in the skull of the European wildcat (Felis silvestris Schreber, 1777). Vertebrate Zoology, 62 (2): 281-294, 2012.

Teixeira, F.S.; Campos, V.; Mitchell, C.; Carvalho, L.M.B. Retenção prolongada de molares decíduos: diagnóstico, etiologia e tratamento. Revista Dental Press de Ortodontia e Ortopedia Facial, 10: 125-137, 2005.

Tutt, C. Small animal dentistry: a manual of techniques. Ames: Blackwell Publishing, 2006. 274p.

Van Nievelt, A.F.H.; Smith, K.K. To replace or not to replace: the significance of reduced functional tooth replacement in marsupial and placental mammals. Paleobiology, 31: 324346, 2005.

Wiggs, R.B.; Bloom, B.C. Dental examination. In: Norsworthy, G.D.; Grace, S.F.; Crystal, M.A.; Tilley, L.P. The feline patient. $4^{\text {th }}$ ed. Iowa: Blackwell Publishing, 2011. p. 597-598. 